\title{
Fisiología del sistema nervioso central y anemia en el paciente neurocrítico posquirúrgico Artículo de revisión
}

Delgado-Guerrero Edgar Gamaliel *, Torres-Soto María de la Luz **, Badenes-Quiles Rafael ***, Belda- Nacher Francisco Javier ${ }^{* * * *}$, Flores Parkman-Sevilla Felipe de Jesús****

\begin{tabular}{|c|c|}
\hline Resumen & Abstract \\
\hline $\begin{array}{l}\text { La comprensión de la fisiología del sistema nervioso se vuelve } \\
\text { indispensable para el análisis y la generación de herramientas } \\
\text { que puedan proporcionar la información fidedigna cuando un } \\
\text { paciente es agredido. Esta revisión tiene como objetivo descri- } \\
\text { bir el funcionamiento general, los procesos de autorregulación } \\
\text { cerebral, realizar una semblanza general de los principales } \\
\text { elementos a considerar en relación a la monitorización de } \\
\text { las variables de repercusión directa sobre el sistema nervioso } \\
\text { central y los criterios generales de acuerdo a consensos sobre } \\
\text { uso de hemoderivados en el paciente con lesiones hacia este } \\
\text { órgano blanco. LUXMÉDICA AÑ̃ 10, NÚMERO 30, MAY0-AGOSTO } \\
\mathbf{2 0 1 5 , P P ~ 3 1 - 4 2}\end{array}$ & $\begin{array}{l}\text { Understanding the physiology of the nervous system becomes } \\
\text { essential for analysis and generation of tools that can provide } \\
\text { the most accurate information when a patient is attacked. This } \\
\text { review aims to describe the general operation, the processes } \\
\text { of cerebral autoregulation, make a general sketch of the main } \\
\text { elements to consider regarding monitoring variables direct im- } \\
\text { pact on the central nervous system and the general criteria } \\
\text { according to consensus on use of blood products in patients } \\
\text { with injuries to the target organ. LUXMÉDICA AÑO 10, NÚMER0 } \\
\text { 30, MAYO-AGOST0 2015, PP 31-42 }\end{array}$ \\
\hline $\begin{array}{l}\text { Palabras Clave: sistema nervioso central, autorregulación, } \\
\text { monitorización neurológica, transfusión sanguínea. }\end{array}$ & $\begin{array}{l}\text { Keywords: nervous central system, self, neurological monito- } \\
\text { ring, blood transfusion. }\end{array}$ \\
\hline
\end{tabular}

\section{Introducción}

A pesar de que la anemia es una de las complicaciones médicas más comunes que se encuentran en los pacientes críticos, la necesidad de glóbulos rojos (RBC) o transfusión de concentrados eritrocitarios en procedimientos neuroquirúrgicos es objeto de debate. ${ }^{1}$ Aunque

* Médico Residente del tercer año del servicio de Anestesiología del Centenario Hospital Miguel Hidalgo Aguascalientes, México.

** Médico adjunto y Secretaria Técnica del posgrado de Anestesiología del Centenario Hospital Miguel Hidalgo.

*** Médico adjunto del Departamento de Anestesiología y Cuidados Intensivos Quirúrgicos del Hospital Clínico Universitario de Valencia, España.

**** Jefe del Departamento de Anestesiología y Cuidados Intensivos Quirúrgicos del Hospital Clínico Universitario de Valencia, España.

***** Jefe de Enseñanza del Centenario Hospital Miguel Hidalgo

Fecha de recibido: 27 de enero 2015

Fecha de aceptación:7 de julio 2015

Correspondencia: Dr Edgar Gamaliel Delgado Guerrero. Departamento de Anestesiología del Centenario Hospital Miguel Hidalgo. Calle Galeana Sur 465 Colonia Obraje. Código postal 20230, Aguascalientes, Ags., México. Teléfono 01(449)9946720 Correo electrónico edgamaliel@hotmail.com 
existen recomendaciones detalladas para muchas otras disciplinas quirúrgicas, los datos son limitados sobre la probabilidad de transfusiones en el perioperatorio de procedimientos neuroquirúrgicos. De acuerdo a los resultados de los ensayos clínicos, las prácticas de transfusión en todo el mundo en general se han vuelto más restrictivas; sin embargo, la entrega reducida de oxígeno contribuye al desarrollo de una lesión cerebral "secundaria", por lo que la anemia puede no ser tan bien tolerada en los pacientes de atención neurocríticos. ${ }^{2}$ De acuerdo a la aseveración anterior, para abordar el tema del paciente neurocrítico es indispensable el conocimiento de conceptos establecidos, de la fisiología, de autorregulación cerebral y de la fisiopatología del paciente con lesión cerebral; así mismo de la compresión de la génesis de la anemia posterior a un procedimiento invasivo, por ello se realiza una descripción detallada de la fisiología del sistema nervioso central y de los elementos de monitorización del paciente con patología neurológica que conlleve al manejo con sangre u otros hemoderivados.

\section{| | | | | | | | | | | | | | | | | | | | | | | | | | | | | | | | | | | | | | | | | | | | | | | | | | | | | | | | | | | | | | | | | | | | | | | | | | | | | | | | | | | | | | | | | | | | | | | | | | | | | ||}

\section{Fisiología y metabolismo cerebral}

La función primaria del cerebro es la generación de una acción en respuesta a la estimulación y esta función es afectada por el movimiento de iones contra gradientes eléctricos y la liberación-regeneración de neurotransmisores en las sinapsis. Estas funciones requieren una gran cantidad de energía en forma de trifosfato de adenosina (ATP). En condiciones normales, el combustible metabólico es la glucosa de manera casi exclusiva y un apropiado suministro de oxígeno para los procesos oxidativos que intervienen. ${ }^{3}$

Medido solo por la masa, el cerebro consume más energía que cualquier otro tejido en el cuerpo. En condiciones basales, aproximadamente el $60 \%$ de esta energía se utiliza para alimentar la bomba de iones-ATPasa, que mantienen los gradientes iónicos a través de membranas neuronales.

La vía metabólica de entrada al cerebro está dada por transporte activo a través de la barrera hematoencefálica, utilizando como transportadores a proteínas denominadas como: GLUT 1 en capilares cere- brales y células del sistema nervioso central (SNC), GLUT 3 a neuronas y GLUT 5 a células microgliales. Estos transportadores de glucosa están regulados en condiciones de hipoxia. La captación de glucosa es alta en el tejido cerebral y la tasa metabólica cerebral de la glucosa (CMRGI) es de aproximadamente $30 \mathrm{mg} / 100 \mathrm{~g} / \mathrm{min}$, lo que representa aproximadamente el $25 \%$ del consumo total de la glucosa. Las reservas energéticas cerebrales en contraste con los requerimientos son limitadas; la hipoglucemia pronto se traduce en disfunción celular cerebral, que se manifiesta clínicamente como ansiedad y confusión, que pronto progresa a convulsiones y coma. Los síntomas observados reflejan la mayor susceptibilidad de las estructuras corticales a la hipoglucemia en comparación con el tronco cerebral. Las células cerebrales contienen glucógeno y toda la glucosa disponible se agota en 2 minutos si el flujo sanguíneo cerebral (FSC) cesa. Después de la absorción en las células cerebrales, aproximadamente el $70 \%$ de la glucosa se oxida a dióxido de carbono y agua; junto con la fosforilación oxidativa dentro de la mito- 
condria, se brinda el ATP necesario para el suministro de energía. El resto se convierte en aminoácidos, proteínas y lípidos. Bajo condiciones de hipoxia los astrocitos metabolizan la glucosa anaeróbicamente por medio de la glucólisis para formar lactato.

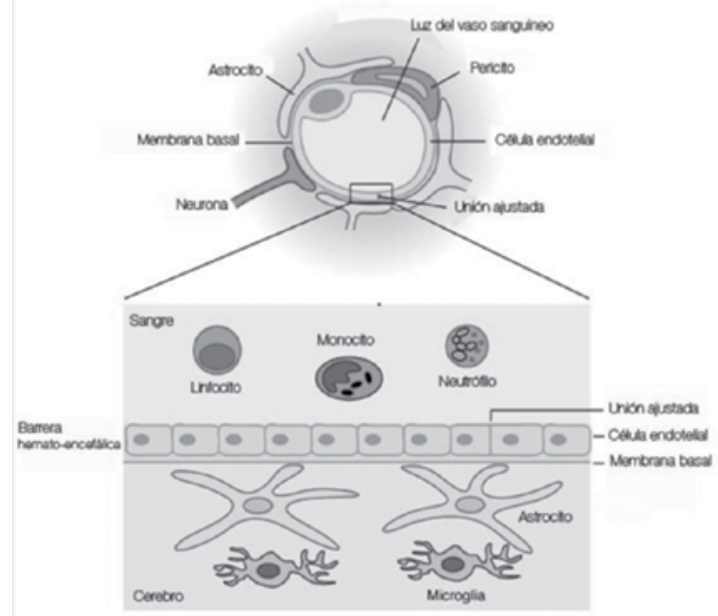

Figura 1. La barrera hematoencefálica. Fuente. Expert Reviews in Molecular Medicine 2003 Cambridge University Press

El metabolismo aeróbico normal a este nivel requiere un abundante suministro ininterrumpido de oxígeno, el cual se obtiene de la sangre arterial. La sangre llega al cerebro a través de las arterias carótidas internas, que posteriormente se emparejan con las arterias vertebrales. Alrededor del $70 \%$ del total del FSC es suministrado por las carótidas. Estas circulaciones, anterior y posterior se unen en el polígono de Willis en la base del cerebro, pero es importante tener en cuenta que esta anastomosis es incompleta hasta en un 50\% de los individuos. ${ }^{4}$

Aunque el cerebro constituye sólo el 2\% del total de masa corporal, recibe el 15\% del gasto cardíaco $(750 \mathrm{ml} / \mathrm{min}$ en adultos). En una situación de reposo, el FSC es de aproximadamente $50 \mathrm{ml} / 100 \mathrm{~g} / \mathrm{min}$, sin embargo, el flujo no se distribuye de manera uniforme. La materia gris, que es metabólicamente más activa, recibe aproximadamente $90 \mathrm{ml} / 100 \mathrm{~g} / \mathrm{min}$ y en estas regiones la tasa de consumo de oxígeno, denominado la tasa metabólica cerebral de oxígeno (CMRO2), es de aproximadamente $3 \mathrm{ml} / 100 \mathrm{~g} / \mathrm{min}$. La materia blanca recibe alrededor de $20 \mathrm{ml} / 100 \mathrm{~g} / \mathrm{min}$ y su $\mathrm{CMRO}_{2}$ es de aproximadamente $1 \mathrm{ml} / 100 \mathrm{~g} / \mathrm{min}$. La interrupción completa del FSC produce pérdida de conciencia en cuestión de segundos al igual que una reducción del FSC aproximadamente de $20 \mathrm{ml} / 100 \mathrm{~g} / \mathrm{min}$. El metabolismo neuronal anaeróbico se produce por debajo de $18 \mathrm{ml} / 100 \mathrm{~g} / \mathrm{min}$ y el electroencefalograma se vuelve plano. La muerte de las células del cerebro (infarto) se lleva a cabo en alrededor de 3 horas con flujos de $10 \mathrm{ml} / 100 \mathrm{~g} / \mathrm{min}$ y después de 30 min a flujos de $5 \mathrm{ml} / 100 \mathrm{~g} / \mathrm{min}$. La presión de perfusión cerebral PPC (es decir, la presión gradiente arteriovenosa) en el cerebro es más compleja que la de otros órganos porque está confinado dentro de una bóveda rígida (tabla 1). Es decir, depende de la diferencia de presión entre la presión arterial media (PAM) y la presión intracraneal (PIC). Esta diferencia de presión se conoce como presión de perfusión cerebral (PPC). Una PPC normal es de 70 a $80 \mathrm{mmHg}$; el umbral para la isquemia crítica es 30 a 40 $\mathrm{mmHg}$. Incluso a los niveles normales de PAM, una PIC elevada de más de $20 \mathrm{mmHg}$ comprometerá la PPC y por lo tanto reducirá el flujo sanguíneo cerebral (2).

\section{Tabla I}

\section{Valores fisiológicos cerebrales normales}

\begin{tabular}{|lc|}
\hline $\begin{array}{l}\text { Flujo sanguíneo } \\
\text { cerebral (FSC) }\end{array}$ & $\begin{array}{c}750 \mathrm{ml} / \mathrm{min} \text { o } 15 \% \text { del } \\
\text { gasto cardiaco }\end{array}$ \\
\hline $\begin{array}{l}\text { Flujo sanguíneo cerebral } \\
\text { global }\end{array}$ & $50 \mathrm{ml} / 100 \mathrm{~g} / \mathrm{min}$ \\
\hline Materia gris & $90 \mathrm{ml} / 100 \mathrm{~g} / \mathrm{min}$ \\
\hline Materia blanca & $20 \mathrm{ml} / 100 \mathrm{~g} / \mathrm{min}$ \\
\hline $\begin{array}{l}\text { Tasa metabólica cerebral } \\
\text { de oxigeno }(\mathrm{CMRO} \\
\text { materia gris }\end{array}$ & $3 \mathrm{ml} / 100 \mathrm{~g} / \mathrm{min}$ \\
\hline $\begin{array}{l}\text { Tasa metabólica cerebral } \\
\text { de oxigeno }\left(\mathrm{CMRO}{ }_{2}\right) \\
\text { materia blanca }\end{array}$ & $1 \mathrm{ml} / 100 \mathrm{~g} / \mathrm{min}$ \\
\hline $\begin{array}{l}\text { Tasa metabólica cerebral } \\
\text { de glucosa }(\mathrm{CMRGl}) \\
\text { total }\end{array}$ & $\begin{array}{c}30 \mathrm{mcg} / 100 \mathrm{~g} / \mathrm{min} \text { o } 25 \% \\
\text { del consumo corporal }\end{array}$ \\
\hline
\end{tabular}


Esto pone de relieve la importancia de mantener una adecuada PAM en circunstancias como traumatismos craneales para asegurar una adecuada perfusión. Las proporciones del contenido del cráneo son: parénquima cerebral (80 \%), sangre (9\%), LCR $(6 \%)$ y el líquido intersticial (5\%). Después de la fusión de las suturas craneales, el cerebro se encuentra dentro de una caja de hueso rígido. La PIC normal es 7 a $12 \mathrm{mmHg}$ y se determina por el equilibrio entre la velocidad de formación de LCR y absorción (este último en función de la presión de los senos venoso y la resistencia de las vellosidades aracnoideas). El valor de la PIC es dinámico, y las fluctuaciones en la presión ocurren con pulsaciones arteriales, posición, la respiración, la tos y el esfuerzo.

La doctrina Monroe-Kellie afirma que debido a los componentes intracraneales el volumen total es fijo, un aumento en el volumen de uno de los componentes contenidos dentro del cráneo, a menos que sea acompañado por una reducción en volumen de los otros componentes, dará lugar a un aumento de la PIC. ${ }^{5}$

En un principio, cuando se produce una lesión, el volumen del cerebro aumenta, entonces se produce una compensación por movimiento de LCR en el compartimiento de la médula, acompañado de un aumento en su absorción y una disminución en la producción, además de una reducción en el volumen sanguíneo cerebral, lo que limita el aumento de la PIC. Sin embargo, cuando se ven abrumados estos mecanismos compensatorios, la distensibilidad intracraneal cae y la PIC aumenta espectacularmente con los más pequeños aumentos en el volumen intracraneal. ${ }^{6}$ Eventualmente, si no se controla, se eleva la PIC y causa compresión del tronco cerebral con hipertensión, bradicardia y respiración irregular (Reflejo de Cushing). ${ }^{7}$

Los anestesiólogos instituyen varios métodos para reducir la PIC de forma aguda en los pacientes de alto riesgo con valores críticamente altos. ${ }^{8}$ Con exclusión de métodos quirúrgicos de drenaje o de descompresión, los procesos para el control de PIC dependerá de la reducción del volumen de sangre intracraneal o del volumen del líquido intersticial. La reducción del volumen sanguíneo intracraneal se puede lograr mediante la reducción de dióxido de carbono arterial ( $\mathrm{PaCO} 2)$, que promueve la vasoconstricción cerebral, aumentando el drenaje venoso con una posición semifowler, es decir, con la cabeza hacia arriba, proporcionando una sedación adecuada y relajación muscular, lo cual reduce la presión intratorácica. La reducción de volumen de fluido intersticial se puede lograr mediante la restricción de líquidos o por la administración de diuréticos (por ejemplo, manitol y furosemida) o corticosteroides. ${ }^{5}$

Por el contrario, si la técnica anestésica es insuficiente, puede resultar en una situación dramática, aumentando la PIC en pacientes con cifras elevadas preexistentes; ${ }^{9-11}$ esta situación ha sido bien estudiada en investigaciones previas. ${ }^{12}$

Con respecto a la dinámica del líquido cefalorraquídeo (LCR) entendemos que es un ultrafiltrado de plasma que circula libremente por todos los ventrículos cerebrales y el canal central de la médula espinal. Es formado a razón de aproximadamente 500 $\mathrm{ml} /$ día en los plexos coroides de los ventrículos laterales y reabsorbido respectivamente en las vellosidades aracnoideas. El LCR fluye entonces a través del foramen de Monroe al tercer ventrículo; en el cuarto ventrículo a través del acueducto de Silvio y luego en la cisterna magna y espacios subaracnoideos a través del agujero medial de Magendie y los orificios laterales de Luschka. En última instancia el LCR es reabsorbido a través las vellosidades subaracnoideas en los senos venosos cerebrales como resultado de un gradiente de presión. ${ }^{3}$

Si la velocidad de formación de LCR supera la tasa de reabsorción (por ejemplo, si la obstrucción de la circulación del LCR 
está presente) ocurre hidrocefalia y resulta en aumento de la PIC. De ahí que cobre importancia especial la existencia de la barrera hematoencefálica la cual previene la transferencia de sustancias potencialmente nocivas que llegan al cerebro. Esta barrera semipermeable consiste en tres capas celulares: el endotelio vascular y su sótano membrana, los astrocitos y los pericitos (Fig.1). ${ }^{13}$ Las células endoteliales carecen de vesículas para realizar pinocitosis y son selladas por uniones estrechas (zona pelúcida occludens) sin espacios anatómicos. La existencia de la barrera hematoencefálica explica la diferencia entre los constituyentes del plasma y el LCR. Las proteínas contenidas en el LCR son muy bajas en comparación con el plasma (0.2 frente a $60 \mathrm{~g} /$ /itro), y un aumento de los niveles proteicos en el LCR indican la interrupción de la barrera. Del mismo modo, las concentraciones de potasio, calcio, glucosa, urea y los linfocitos son más bajos en LCR. ${ }^{14}$ El paso de sustancias a través de la barrera hematoencefálica es directamente proporcional a su solubilidad en los lípidos y está facilitado por mecanismos de transporte activo, pero es inversamente proporcional al peso molecular, carga iónica y la cantidad de proteínas plasmáticas de unión. Las sustancias lipófilicas (dióxido de carbono, oxígeno y agentes anestésicos entre otros) pasan libremente, a diferencia de las sustancias con gran peso molecular (proteínas) y elementos con alta carga iónica (sodio). Las proteínas y fármacos (penicilina) no pueden cruzar la barrera a menos que exista un proceso inflamatorio (meningitis). La integridad de la barrera puede ser examinada por la inyección intravenosa de material radiactivo, isótopos unidos a proteínas; las técnicas de exploración pueden entonces ser utilizados para determinar si el marcador radiactivo se escapa de vasos cerebrales. Algunos aneurismas rotos o el aumento de la permeabilidad vascular en sitios donde exista un tumor, se pueden detectar usando tales técnicas. ${ }^{15}$ El agua se mueve libremente a través de la barrera hematoencefálica, dependiendo de gradientes osmóticos. Los cambios bruscos de osmolaridad plasmática secundario a los cambios en las concentraciones de glucosa o electrolitos pueden, por lo tanto, conducir a cambios potencialmente problemáticos en el cerebro. ${ }^{16}$ Esto es un punto importante de la corrección de sodio y glucosa. La barrera hematoencefálica se puede irrumpir mediante varios procesos, incluyendo hipertensión, accidente cerebrovascular, traumas, estado epiléptico, hipercapnia, hipoxia y especialmente los procesos inflamatorios (química, infecciosa o autoinmune). Cuando ocurre irrupción de la membrana, el movimiento del fluido se realiza dependiente de gradientes hidrostáticos. ${ }^{17}$

Respecto al control de flujo sanguíneo cerebral, existen varios mecanismos que permiten que a nivel basal se pueda garantizar el suministro y las demandas de sustrato metabólico del cerebro, además de los mecanismos de regulación locales que dirigen el flujo de sangre a las regiones del cerebro que son particularmente activos (es decir, el flujo de sangre está acoplado a necesidades metabólicas locales). Entonces esta asociación del metabolismo de flujo y de reguladores químicos locales provoca un aumento en el $\mathrm{CMRO}_{2}$ y CMRGI que se acompaña de un aumento del FSC regional, para que la entrega de glucosa y el oxígeno sean garantizados. El cambio paralelo en el FSC con $\mathrm{CMRO}_{2}$ y CMRGI se conoce como acoplamiento metabolismo de flujo. ${ }^{18}$

Hay pruebas que sugieren que el FSC puede ser modulado por los cambios en el consumo de glucosa, más que el consumo de oxígeno bajo condiciones de hipoxia. Los cambios regulatorios involucrados en el acoplamiento del metabolismo de flujo pueden tener una latencia corta (aproximadamente 1 segundo), donde los cambios transitorios en la concentración de 
mediadores locales metabólicos parecen determinar la falta de control de FSC regional al afectar el tono vascular. ${ }^{18,19}$

La vasoconstricción se produce por la acción de los iones de calcio libre, tromboxano (un producto de ácido araquidónico) y endotelina (secretada por las células endoteliales del músculo liso vascular). Algunos fármacos antagonistas del calcio pueden producir vasodilatación y prevenir la liberación de adenosina. Las sustancias vasodilatadores potentes a este nivel incluyen: el potasio perivascular (lanzado en altas concentraciones durante estados convulsivos, hipoxia, y la estimulación eléctrica), la adenosina, un metabolito del ATP (en respuesta a la hipotensión arterial y la hipoxia), así como prostaglandinas (por ejemplo, $\mathrm{PGE}_{2}$ y prostaciclina $\mathrm{PGI}_{2}$ ), lactato, la acetilcolina, la serotonina, la sustancia $\mathrm{P}$ y el óxido nítrico. El óxido nítrico es sintetizado por las células endoteliales y luego se difunde en la capa de músculo liso por inducción de guanosina cíclico monofosfato (GMP), lo que lleva al músculo liso a la relajación y por lo tanto vasodilatación de los vasos cerebrales. ${ }^{20}$

Autorregulación. La autorregulación es el mantenimiento del FSC constante a pesar de variaciones en PPC. En condiciones normales, cuando la PIC y la PPC son bajos, la presión de perfusión arterial sistémica (PAM) se convierte en el principal determinante de la PPC. En un rango de $50 \mathrm{mmHg}$ y $150 \mathrm{mmHg}$ de presión arterial el FCS se mantiene constante $(50 \mathrm{ml} / 100 \mathrm{~g} /$ min) Figura 2. Sin embargo, la autorregulación tiene sus límites fisiológicos, el FSC está directamente relacionado con la presión de perfusión. Cuando existen alteraciones en la resistencia vascular cerebral (RVC); después 10 a 60 segundos, se produce modificación de la tensión transmural en los vasos de resistencia; por lo tanto, a medida que aumenta la PPC de 50 a 150 $\mathrm{mmHg}$, las arteriolas cerebrales se constriñen y producen restricción en los aumentos del FSC. ${ }^{21}$

La autorregulación puede ser modificada por la actividad del sistema nervioso

AUTORREGULACIÓN CEREBRAL

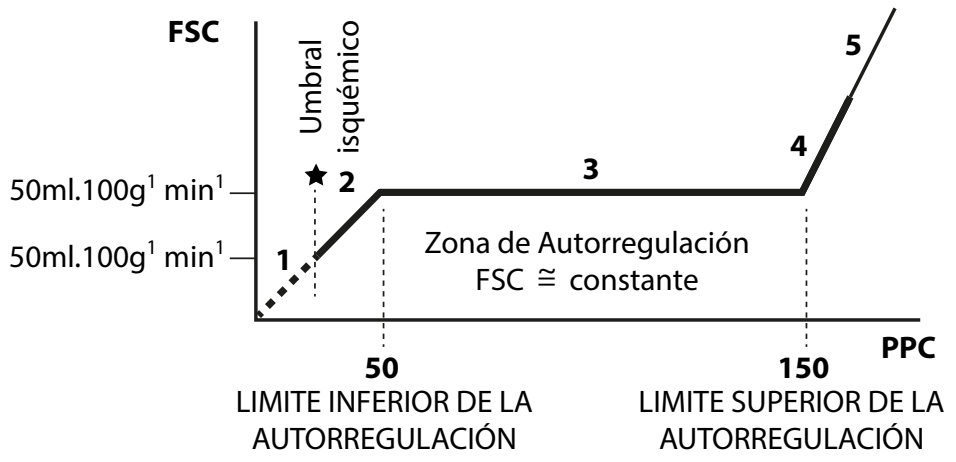

Figura 2. Gráfica de autorregulación cerebral. Fuente. Gómez Luciano Ariel. Bases fisiopatológicas de la neuroanestesia Rev.Arg. Anest. 1998; 56: 4: 211-224

simpático. Por lo tanto, la hipertensión crónica o la estimulación desplazan la curva de autorregulación a la derecha, mientras que el bloqueo simpático o simpatectomía cervical desplaza la curva a la izquierda. Los síntomas de la isquemia se producen sólo cuando la PAM cae por debajo de $60 \%$ del límite inferior de autorregulación. Por arriba del límite superior de autorregulación, mecanismos como la dilatación forza- 
da de las arteriolas cerebrales, la inversión de los gradientes hidrostáticos y el edema cerebral, dan como resultado incrementos del volumen sanguíneo cerebral y de PIC. La autorregulación se interrumpe en presencia de obstrucciones intracraneales, hipoxemia, hipercapnia vascular fija (por ejemplo, de ateroma carotídeo) e inclusive el uso de agentes anestésicos volátiles. ${ }^{22}$

Aumento de la presión venosa cerebral. Una presión venosa cerebral elevada reduce el drenaje venoso cerebral, dado que se expande el volumen sanguíneo cerebral, se interrumpen las fuerzas de Starling a nivel capilar que conducen a edema cerebral, esto se traduce en un aumento de la presión intracraneal y por lo tanto reduce el FSC. Puede ser causado por obstrucción del drenaje venoso por la compresión del cuello (collarines o lazos, tubo traqueal), de posicionamiento de la cabeza hacia abajo (por ejemplo, durante la inserción de una vía central) o de un aumento de la presión intratorácica (por ejemplo, al toser, hacer esfuerzo, relajación muscular insuficiente y la aplicación de presión positiva al final de la espiración PEEP, durante la ventilación con presión positiva). ${ }^{23}$

La tensión arterial de dióxido de carbono. El dióxido de carbono es un potente vasodilatador de los vasos sanguíneos cerebrales. Cuando la $\mathrm{PaCO}_{2}$ se eleva entre $3,5 \mathrm{kPa}(26 \mathrm{mmHg})$ y $8 \mathrm{kPa}(60 \mathrm{mmHg})$ hay un aumento lineal en el FSC. Por arriba de una $\mathrm{PaCO}_{2}$ de $60 \mathrm{mmHg}$ los vasos cerebrales sufren una dilatación máxima y no es posible un mayor aumento en su diámetro, por el contrario, en una $\mathrm{PaCO}_{2}$ de 3 $\mathrm{kPa}$, los vasos cerebrales son máximamente constreñidos. El efecto de la hipocapnia en la vasculatura cerebral se consigue aumentando la concentración de iones de hidrógeno cerebrales. El efecto vasoconstrictor de una $\mathrm{PaCO}_{2}$ baja se atenúa progresivamente por una caída en el nivel de bicarbonato del cerebro, que normaliza el $\mathrm{pH}^{24}$
Tensión arterial de oxígeno y el contenido de oxígeno. El FSC es directamente sensible a los cambios en el suministro de oxígeno y permanece inalterado hasta que un umbral de una tensión arterial de oxígeno $\left(\mathrm{PaO}_{2}\right)$ de $6,8 \mathrm{kPa}(50 \mathrm{mmHg})$ se alcanza. Por debajo de este umbral, el FSC se eleva drásticamente. Esto corresponde a la parte empinada de la curva de disociación de oxihemoglobina, es decir, el FSC es sensible a la $\mathrm{PaO}_{2}$ pero no al contenido de oxígeno. Este efecto explica el modesto aumento del FSC $(10 \%)$ cuando se respira al $100 \%$ de oxígeno. ${ }^{25}$

Hematocrito. El hematocrito es el principal determinante de la viscosidad de la sangre y del contenido de oxígeno (y por lo tanto el suministro), cambios del FSC están inversamente relacionados con la viscosidad de la sangre. Dentro de la gama normal de hematocrito, esto tiene un efecto mínimo en el FSC. Sin embargo, en ciertas situaciones en las que el FSC se disminuye patológicamente (vasoespasmo cerebral tras una hemorragia subaracnoidea), una disminución en el hematocrito por hemodilución puede mejorar el FSC. ${ }^{26}$

\section{Medición del flujo sanguíneo cerebral}

Un número importante de técnicas para la medición del FSC han surgido desde el método pionero de Kety y Schmidt en 1945. Muchas técnicas permiten ahora a la medición del flujo sanguíneo regional, proporcionando información útil sobre los cambios en el flujo sanguíneo en las partes enfermas del cerebro. ${ }^{27}$

Técnica KetyeSchmidt. ${ }^{28}$ El principio de Fick establece que el flujo de sangre a través de un órgano se puede medir mediante la determinación de la cantidad de un gas inerte (sustancia Q) que se retira de la circulación sanguínea por el órgano por unidad de tiempo, y divide ese valor por la diferencia entre la concentración de la sustancia en la sangre arterial $[\mathrm{A}]$ y la 
concentración en la sangre venosa [V] en el órgano FSC $1 / 4 Q^{1 / 2} A 1 / 2 \mathrm{~V}$.

Técnica de lavado de Xenón ${ }^{133}$. El flujo sanguíneo cortical regional se puede medir mediante el control de eliminación del isótopo radiactivo inhalado xenón ${ }^{133}$ utilizando una pieza de contadores de centelleo colocados sobre la cabeza. La pendiente de la curva a lavar de salida del trazador radiactivo es proporcional al FSC bajo en el transductor. La técnica no proporciona un análisis del FSC regional sino que evalúa principalmente el flujo cortical de sangre. La resolución tridimensional se puede lograr utilizando una reconstrucción en tomografía computarizada en una técnica llamada de emisión de fotón único. ${ }^{29,30}$

La ecografía Doppler transcraneal (TCD). ${ }^{31}$ Implica la aplicación de un campo de baja frecuencia $(2 \mathrm{MHz})$ con un haz de ultrasonido a la ventana transtemporal delgada deshuesada, lo que permite la evaluación de la parte media y las arterias cerebrales anteriores. Las velocidades de flujo dentro de estos vasos se pueden determinar mediante el efecto Doppler. Si el ángulo del haz de ultrasonido y el diámetro del vaso se mantienen constantes, los cambios relativos en el flujo y velocidad se correlacionan estrechamente con los cambios en el FSC.

La medición de la oxigenación cerebral.,25,32 Oximetría del bulbo yugular. El bulbo yugular es una dilatación de la vena yugular interna por debajo de la base del cráneo, utilizando un catéter mediante técnica de Seldinger. Se toma una muestra de sangre para medir la tensión de oxígeno y la saturación, dando un reflejo del FSC. Valores más bajos refleja una mayor captación por el cerebro y por lo tanto menos flujo sanguíneo, suponiendo que el consumo de $\mathrm{O}_{2}$ permanece constante. La importante desventaja de esta técnica es que el FSC global solo puede interpretar cambios estimados y no regionales. Además, si el FSC y el consumo de oxígeno disminuyen demasiado (por ejemplo, en una lesión cerebral grave), la saturación venosa yugular puede no modificarse.

Microdiálisis intracerebral. Esta técnica consiste en la inserción de un catéter fino, que contiene una membrana para diálisis de perfusión con solución Ringer en el parénquima cerebral. Las moléculas que participan en las vías metabólicas cerebrales son controladas en forma directa y esto puede revelar información a la adecuación de la oxigenación cerebral y el flujo sanguíneo. Metabolitos tales como glucosa, piruvato, lactato, glutamato y de glicerol o drogas (fenitoína) que se difunden en la solución de la sonda del líquido intersticial (espacio extracelular) a través de la membrana, se pueden analizar. El lactatopiruvato refleja la disponibilidad de oxígeno cerebral regional, y se ha utilizado clínicamente en la investigación de lesiones en la cabeza y la hemorragia subaracnoidea. Un aumento de la tasa sugiere que el metabolismo anaeróbico está produciendo flujo sanguíneo cerebral regional insuficiente (secundaria a la hipoxia); entonces se puede iniciar tratamiento para corregir esta fisiología alterada. ${ }^{33}$

Presión parcial de oxígeno cerebral. ${ }^{34}$ Los sensores pueden ser insertados en el parénquima cerebral para medir la presión parcial de oxígeno en el fluido extra-celular del cerebro $\left(\mathrm{pBRO}_{2}\right)$, lo que refleja la disponibilidad de oxígeno para metabolismo oxidativo. Los valores obtenidos reflejan en general el balance entre la entrega y el consumo de oxígeno. Esta técnica está siendo utilizada para optimizar el tratamiento de la hemorragia subaracnoidea y lesión cerebral traumática. Esta técnica de monitorización se basa en el principio de que la luz con longitudes de onda en la región infrarroja (650 a $900 \mathrm{~nm}$ ) se transmite a través de los tejidos biológicos, y se está convirtiendo en un elemento cada vez más utilizado para eventos en la corteza cerebral. Los fotones producidos por un foto- 
diodo láser se dirigen al cráneo, mientras algunos se reflejan y se dispersan, otros se transmiten. Ciertos compuestos coloreados dentro de los tejidos (cromófobos), especialmente la oxihemoglobina, desoxihemoglobina y la enzima citocromo oxidasa, tienen características de espectros de absorción. La intensidad de la luz emergente se detecta y una computadora convierte los cambios en la intensidad de la luz en cambios en la concentración de cromóforo. Las aplicaciones clínicas de esta técnica incluyen el monitoreo de la oxigenación cerebral, FSC y el volumen.

\section{Anemia en el paciente neurocrítico posquirúrgico}

La anemia es definida por la Organización Mundial de la Salud como la concentración de hemoglobina $(\mathrm{Hb})$ inferior a $12 \mathrm{~g} / \mathrm{dl}$ en mujeres y $13 \mathrm{~g} / \mathrm{dl}$ en los hombres. ${ }^{8}$ Es uno de los trastornos médicos más comunes encontrados en los pacientes críticos, con trastornos neurológicos. Alrededor de dos tercios de los pacientes tienen concentraciones de $\mathrm{Hb}$ menores de $12 \mathrm{~g} / \mathrm{dl}$ en el momento de ingresar a la Unidad de Cuidados Intensivos ( $\mathrm{UCl}$ ), con un decremento posterior de alrededor de $0.5 \mathrm{~g} / \mathrm{dl}$ por día. La etiología de la anemia adquirida en la $\mathrm{UCl}$ es multifactorial.

La anemia es frecuente después de una lesión cerebral traumática, se produce en hasta $50 \%$ de los pacientes. Normalmente, la dilatación de las arteriolas cerebrales aumenta el FSC y preserva el oxígeno en la zona con menor contenido, por lo tanto, los síntomas de la disfunción cerebral inducida por la anemia se ponen de manifiesto sólo cuando la hemoglobina $(\mathrm{Hb})$ es menos de $7 \mathrm{~g} / \mathrm{dl}$. En condiciones de deterioro cerebral, como ocurre después del trauma craneal, los mecanismos compensatorios pueden ser insuficientes para mantener un FSC adecuado y la lesión cerebral inducida por la anemia puede ocurrir con umbrales más altos de $\mathrm{Hb}$, por ejemplo 9-10g/dl . En modelos animales de lesión cerebral traumática, la anemia reduce la oxigenación cerebral y agrava la lesión cerebral secundaria. Aunque la relación entre la anemia y el pronóstico del traumatismo craneal sigue siendo controvertida, se han realizado algunos estudios que pueden sugerir por medio de metas los niveles de hemoglobina y hematocrito traspolados a pacientes con características similares. ${ }^{35-37}$ Además, la corrección de la anemia con la transfusión de glóbulos rojos, particularmente cuando se busca un nivel de $\mathrm{Hb}$ mayor que $10 \mathrm{~g} / \mathrm{dl}$, puede estar asociado con una mayor morbilidad. Las directrices recomiendan que la anemia no deben ser el único factor que se utiliza para decidir si se debe administrar transfusiones, sin embargo, no hay marcadores fisiológicos establecidos para guiar la toma de decisiones en el trauma craneal de pacientes con anemia.

Aunque las concentraciones de hemoglobina tan bajas como $7 \mathrm{~g} / \mathrm{dl}$ son bien toleradas en pacientes críticamente enfermos, tal grado severo de anemia podría ser perjudicial en pacientes con lesión cerebral. Son necesarios ensayos aleatorios de diferentes umbrales de transfusión, especialmente en los entornos de atención de pacientes neurocríticos. ${ }^{38}$ El impacto de la duración de almacenamiento de sangre en las implicaciones neurológicas de transfusión también requiere una mayor investigación. Estudios clínicos observacionales en medicina perioperatoria han demostrado un aumento progresivo en la mortalidad que es proporcional a tanto la anemia preoperatoria crónica y aguda. Sin embargo, este conocimiento aún no ha ayudado a definir el umbral crítico para $\mathrm{Hb}$, la lesión de órganos y la mortalidad en poblaciones específicas de pacientes o en pacientes individuales.

Si bien se debe evitar la hipovolemia, la hipervolemia confiere algunos beneficios adicionales. ${ }^{38}$ En general, la hipertensión se asocia a un mayor flujo sanguíneo ce- 
rebral, independientemente del estado del volumen (normo o hipervolemia).

3,026 pacientes adultos sometidos a procedimientos neuroquirúrgicos en el Hospital Universitario de Saarland, Alemania, entre diciembre de 2006 y junio de 2008 a los que se les transfundieron glóbulos rojos se analizaron retrospectivamente. En general, la probabilidad de transfusión en procedimientos de neurocirugía fue de $1.7 \%$. De ésta, la probabilidad fue de $6.5 \%$ para el hematoma subdural agudo, $6.2 \%$ para tumores de columna vertebral, $4.6 \%$ para la hemorragia intracerebral, $2.8 \%$ para absceso, $2.4 \%$ para lesiones cerebrales traumáticas, $2.3 \%$ para la isquemia cerebral, $1.9 \%$ para hemorragia subaracnoidea/aneurismas, un $1.4 \%$ para los tumores cerebrales, $0.8 \%$ para la hidrocefalia, $0.4 \%$ para las enfermedades degenerativas de la columna vertebral, incluyendo $3.6 \%$ para la fusión intersomática lumbar posterior y del $0 \%$ para el hematoma epidural.

Un paradigma esencial en la gestión de la atención de pacientes neurocríticos es evitar daño cerebral "secundario". La lesión aguda cerebral es vulnerable a trastornos sistémicos, tales como hipotensión, hipoxemia o la fiebre, que puede exacerbar aún más el daño neuronal. Por lo tanto, el cuidado crítico se debe focalizar en intentar mantener un medio fisiológico que minimice la lesión secundaria, ofreciendo así la posibilidad de una recuperación funcional y un estado neurocognitivo favorable.

La pérdida de sangre se acelera por flebotomías frecuentes, y hemorragia ocasional. Los grandes volúmenes de fluido utilizado durante la reanimación, con hemodilución resultante, también puede contribuir a la reducción en los niveles de hemoglobina. La proporción de pacientes que reciben sangre durante su estancia en la UCl varía de $20 \%$ a $44 \%$, y los que se transfunden reciben un promedio de hasta cinco unidades. Sin embargo, en dos estudios multicéntricos, aleatorizados y controlados (ECA) $)^{35,39}$ y dos grandes estudios observacionales, se demuestra que el uso liberal de las transfusiones de sangre, con el objetivo de mantener las concentraciones de $\mathrm{Hb}$ relativamente arbitrarias (por ejemplo, $10 \mathrm{~g} / \mathrm{dl}$ ), han arrojado no sólo ser ineficaces en la mejora de los resultados, sino también potencialmente dañinos. ${ }^{35}$ Sin embargo, la teoría de que la disminución en la entrega de oxígeno (O2) es un factor importante en la lesión cerebral secundaria, sigue siendo incierto; estos hallazgos pueden ser ampliamente aplicados a los pacientes de atención neurocríticos. En consecuencia sigue siendo una práctica común para los médicos establecer metas de niveles de $\mathrm{Hb}$ en un mínimo de 9 a 10 $\mathrm{g} / \mathrm{dl}$ en este contexto. Se han hecho búsquedas de hasta 2137 publicaciones dirigidas principalmente a los adultos ( $>18$ años). ${ }^{37}$ Artículos de revisión relevante, informes de casos y referencias de trabajos seleccionados también han sido tomados en cuenta para generar líneas que involucran a grupos específicos de atención del paciente neurocrítico.

\section{Conclusiones}

La probabilidad de transfusión de sangre durante procedimientos neuroquirúrgicos es muy por debajo del $10 \%$, lo que generalmente se define como el límite para la reserva preoperatoria de los glóbulos rojos. Los pacientes con tumores de la médula, 
hematoma subdural agudo o hemorragia intracraneal, es decir, los pacientes sometidos a grandes procedimientos descompresivos de hueso o tejido blando, tendrán una mayor probabilidad de transfusión. El nivel exacto en el que la anemia amenaza la oxigenación tisular es desconocido y muy probablemente varía según el tipo de tejido y patología. Se ha demostrado que la hemodilución aguda hasta $5 \mathrm{~g} / \mathrm{dl}$ en voluntarios sanos no hace comprometer la tensión de oxígeno del tejido subcutáneo debido a compensación al aumentar el flujo sanguíneo. Sin embargo, la entrega de oxígeno reducida contribuye a una lesión cerebral "secundaria", por lo que la anemia bajo estos métodos puede no ser tan bien tolerada en pacientes neuroquirúrgicos. Aunque las concentraciones de hemoglobina tan bajas como $7 \mathrm{~g} / \mathrm{dl}$ son bien toleradas en pacientes de atención crítica, tal grado severo de anemia podría ser perjudicial en pacientes con lesión cerebral.

No se han realizado ensayos controlados aleatorios que evalúen adecuadamente los umbrales óptimos de transfusión específicamente entre los pacientes con lesiones cerebrales. La importancia de la isquemia y las implicaciones de la anemia no son necesariamente las mismas para todas las condiciones del cuidado neurocrítico. Sin embargo, existe un amplio cuerpo de trabajo experimental, así como estudios observacionales que han avanzado en el conocimiento de esta área y que podrán proporcionar alguna orientación a los clínicos. Las concentraciones de hemoglobina más bajas se asocian consistentemente con los parámetros fisiológicos peores y los resultados clínicos, sin embargo, esta relación no puede ser alterada por un uso más agresivo de transfusiones de glóbulos rojos. El impacto de la duración del almacenamiento de sangre en las implicaciones neurológicas de pacientes neuroquirúricos también requiere una mayor investigación.

\section{Bibliografía}

1. Liberal or restrictive transfusion in high-risk patients after hip surgery. Carson JL, Terrin ML, ,et al. 2011, N Engl J Med 2011, Vol. 365, págs. 2453-2462.

2. Applied cerebral physiology. Taylor, Christopher. 2010, Neurosurgical anaesthesia anaesthesia and intensive care medicine, págs. 11-19.

3. Barrett $K E$, Barman $S M$, Boitano $S$, Brooks $H$. Ganong's review of medical physiology. 23rd edn. New York: McGraw Hill Medical, 2009.

4. Edvinsson L, Krause DN, eds. Cerebral blood flow and metabolism. Philadelphia : Lippincott, Williams and Wilkins, 2002.

5. The Monro-Kellie hypothesis: applications in CSF volume depletion. B., Mokri. 12, 2001, Neurology, Vol. 56, págs. 1746-1748.

6. The new ICP minimally invasive method shows that the Monro-Kellie doctrine is not valid. Mascarenhas S1, Vilela GH, Carlotti C, Damiano LE, Seluque W, Colli B, Tanaka K, Wang CC, Nonaka KO. 2012, Acta Neurochir Suppl, Vol. 114, págs. 117-120.
7. History of the Cushing reflex. Fodstad, Harald M.D., Ph.D., Kelly, Patrick J. M.D. y Buchfelder, Michael M.D. 5, 2006, Neurosurgery, Vol. 59, págs. 11321137.

8. Cerebral blood flow and intracranial pressure. Shardlow, Emily. 5, 2008, Anaesthesia Journal, Vol. 9, págs. 222-225.

9. Controversies in the Anesthetic Management of Intraoperative Rupture of Intracranial Aneurysm. Tumul Chowdhury, Andrea Petropolis, Marshall WiIkinson, Bernhard Schaller, Nora Sandu, and Ronald B. Cappellani. 2014, Anesthesiology Research and Practice, págs. 110-120.

10. Anaesthetic-related neuroprotection. Schifilliti, G. Grasso, A. Conti, and V. Fodale. 11, 2010, CNS Drugs, Vol. 24, págs. 893-907.

11. Influence of anesthesia on cerebral blood flow, cerebral metabolic rate, and brain functional connectivity. al, Vincent Bonhomme Et. 5, 2011, Current opinion in anaesthesiology, Vol. 24, págs. 474-479.

12. Cerebral blood flow change during volatile induction in large-dose sevoflurane versus intravenous propo- 
fol induction: transcranial Doppler study. Hwa Sung Jung, Tae-Yun Sung, Hyun Kang, Jin Sun Kim, TaeYop Kim. 5, 2014, Korean J Anesthesiol. , Vol. 67, págs. 323-328.

13. Pericyte function in the physiological central nervous system. Muramatsu R, Yamashita T. 2014, Neurosci Res, págs. 38-41.

14. 10 most commonly asked questions about cerebrospinal fluid characteristics in demyelinating disorders of the central nervous system. Mehling $M$, Kuhle J, Regeniter A. 2008, Neurologist, págs. 14-60.

15. M., Brooks. Serial Screening for Cerebral Aneurysm Fruitful. Medscape Medical News. [En línea] 14 de May de 2014. [Citado el: 7 de Ene de 2015.] http:// www.medscape.com/viewarticle/824618..

16. Glucose and the injured brain-monitored in the neurointensive care unit. Rostami, Elham. 2014, Front. Neurol, págs. 75-86.

17. Monitoring of cerebral blood flow and metabolism bedside in patients with subarachnoid hemorrhage a Xenon-CT and microdialysis study. imageEHenrik Engquist, Ulf Johnson, Timothy Howells, Elisabeth Ronne-Engström. 2014, Front. Neurol, págs. 345355.

18. Autophagy in the physiology and pathology of the central nervous system. Nikoletopoulou V, Papandreou M, Tavernarakis N. 2014, Cell Death Differ. 2014 Dec 19. doi: 10.1038/cdd.2014.204, págs. 204-213.

19. The Diagnostic Usefulness of Cerebrospinal Fluid Lactic Acid Levels in Central Nervous System Infections. Cunha, Burke A. 8, 2004, Clin Infect Dis, Vol. 39, págs. 1260-1261. .

20. The role of inflammation in CNS injury and disease. SM Lucas, NJ Rothwell. 1, 2006, The british journal of pharmacology, Vol. 147, págs. 232-240.

21. Dynamic Cerebral Autoregulation in Acute Lacunar and Middle Cerebral Artery Territory Ischemic Stroke. Rogier V. Immink, MD y Gert A. van Montfrans, MD. 2005, Stroke, Vol. 36, págs. 2595-2600.

22. Cerebral autoregulation and anesthesia. Dagal, Armagan y Lam, Arthur M. 5, 2009, Current Opinion in Anaesthesiology, Vol. 22, págs. 547-552.

23. Effects of positive end-expiratory pressure on regional cerebral blood flow, intracranial pressure, and brain tissue oxygenation. Muench, Elke MD, y otros. 10, 2005, Critical Care Medicine, Vol. 33, págs. 2367-2372.

24. Ventilatory, cerebrovascular, and cardiovascular interactions in acute hypoxia: regulation by carbon dioxide. Philip N. Ainslie, Marc J. Poulin. 1, 2004, Journal of Applied Physiology, Vol. 97, págs. 149159 .

25. Evidencias en la utilidad de la saturación yugular de oxígeno como método de neuromonitoreo y guía de tratamiento. LG Scalise, LA Camputaro. 2014, REV ARGENT NEUROC, págs. 135-148.

26. Hemoglobin, hematocrit and changes in cerebral blood flow: The SMART-MR study. al., Pieternella $\mathrm{H}$. van der Veen Et. 2014, Neurobiolaging, págs. 210222.
27. Neuromonitoring in Intensive Care: A New Brain Tissue Probe for Combined Monitoring of Intracranial Pressure (ICP) Cerebral Blood Flow (CBF) and Oxygenation. E. Keller, Froehlich, Sikorski. 2, 2011, Acta Neurochirurgica Supplements Volume 110/2, 2011, pp , Vol. 110, págs. 217-220

28. The Kety-Schmidt Technique for Quantitative Perfusion and Oxygen Metabolism Measurements in the MR Imaging Environment. J.J. Leea, W. J. Powers. 2013, AJNR, págs. 100-114.

29. Techniques of Intraoperative Cerebral Blood Flow Measurement. Jonathan A. Friedman, M.D., Robert E. Anderson, B.S., Fredric B. Meyer, M.D. 2000, Neurosurg Focus, págs. 195-215.

30. Regional cerebral blood flow studied by Xenon133 : intra-arterial injection studies and inhalation studies using emission tomography. Lassen, Niels A. 2015, Bulletin der Schweizerischen Akademie der Medizinischen Wissenschaften, págs. 93-100.

31. Transcranial Doppler and anesthetics. V., Fodale. 7, 2007, Acta Anaesthesiologica Scandinavica (Impact Factor: 2.31). 09/2007; 51(7):839-47. DOI: 10.1111/j.1399-6576.2007.01355, Vol. 51, págs 839-847.

32. Comparison of MRI methods for measuring wholebrain venous oxygen saturation. Suliman Barhoum, Zachary B. Rodgers, Michael Langham, Jeremy F. Magland, Cheng Li and Felix W. Wehrli. 2014, Magnetic Resonance in Medicine, págs. 314-323.

33. Cerebral extracellular chemistry and outcome following traumatic brain injury: a microdialysis study of 223 patients. Ivan Timofeev, Keri L. H. Carpenter, Jürgens Nortje, Pippa G. Al-Rawi, Mark T. O'Connell, Marek Czosnyka, Peter Smielewski, John D. Pickard, David K. Menon, Peter J. Kirkpatrick, Arun K. Gupta, Peter J. Hutchinson. 2011, Brain, págs. 484-494.

34. Use of Central Venous Oxygen Saturation to Guide Therapy. Walley, Keith R. 11, 2011, American Journal of Respiratory and Critical Care Medicine, Vol. 5, págs. 514-520.

35. Anemia and red blood cell transfusion in neurocritical care. Kramer AH, Zygun DA. 2009, Crit Care, págs. 13-23.

36. The CRIT Study: Anemia and blood transfusion in the critically ill-current clinical practice in the United State. Corwin HL, Gettinger A, Pearl RG, et al. 2004, Crit Care Med, Vol. 32, págs. 39-52.

37. Clinical practice guideline: red blood cell transfusion in adult trauma and critical care. Napolitano LM, Kurek S, Luchette FA, et al. 2009, Crit Care Med, págs. 3124-3157.

38. Liberal or restrictive transfusion in high-risk patients after hip surgery. Carson JL, Terrin ML, Noveck H, et al. 2011, N Engl J Med, Vol. 365, págs. 2453-2462.

39. MA. A high ratio of plasma and platelets to packed red blood cells in the first 6 hours of massive transfusion improves outcomes in a large multicenter. Zink KA, Sambasivan CN, Holcomb JB, Chisholm G, Schreiber. 2009, Am J Surg, Vol. 197, págs. 565570. 